\title{
How Do Suppliers Benefit from Assessment of Consumer Needs
}

\author{
Ruoyue Zhang* \\ Business School of Xian International Studies University, Xian, China \\ *Corresponding author. Email: 540243948@qq.com
}

\begin{abstract}
Today's economies in the world have shown the characteristics of high debt, low interest rates, low inflation and low growth in recent years. In 2020, due to the impact of trade protectionism and the global epidemic, the uncertainty and downward pressure on economic growth will further increase. Under the circumstances, online consumption is becoming more and more popular, and the digital transformation of the real economy is also accelerating, so consumers are more cautious in purchasing decisions. In this situation, business operations are becoming more and more difficult. This article, based on Professor Richard Taylor's Nudge Theory, combines with the current market situation and consumer psychology, and finds that the ingeniously designed environment has formed an invisible and powerful impetus to make consumers have better choices in a free state. Meanwhile, this action is conducive to satisfying consumer needs, greatly improving consumer satisfaction, enhancing the company's market competitiveness, and helping conpamies to realize their ultimate goals.
\end{abstract}

Keywords: Nudge Theory, Consumer Psychology, Consumer Needs.

\section{供应商如何从消费者需求评估中获益}

\author{
张若月*
}

西安外国语大学商学院, 西安, 中国

*通讯作者. 邮箱: 540243948@qq.com

\section{中文摘要}

当今世界主要经济体近年来呈现高负债、低利率、低通胀和低增长的特点。2020 年, 全球经济因为受到贸易 保护主义和全球疫情的冲击, 经济增长的不确定性和下行压力进一步加大。在此环境下, 线上消费越来越显著, 实体经济数字化转型也不断加快，而消费者的购买决策则相对更加谨慎。新的经济形势下，企业的经营越来越 困难。本文借助诺贝尔经济学奖得主理查德.泰勒教授的助推理论, 结合市场现状及消费心理探究发现可以通 过借用助推理论巧妙设计的环境在自由的状态下对消费行为形成无形且强大的推力，帮助人们做出更好的选 择，同时此举有利于满足消费者需求，大幅提升消费者满意度，增强企业的市场竞争力，促使企业的最终目标 得以有效实现。

关键词: 助推理论, 消费心理, 消费者需求

助推理论是 2008 年由芝加哥大学行为经济学家理 查德. 泰勒和哈佛大学法学家卡斯. 桑斯坦提出来的。
泰勒也是第一位将心理学融入经济学中并衍生出行 为经济学这一学科的学者。该理论是从 Nudge 直译过 
来的，意思是用胳膊肘轻推一下，给人轻轻一击提个 醒。由此可见, 助推是看似不经意的, 轻微的提醒, 是用一种可预见性的方式悄悄地去改变人们的选择 或者改变他们的动机及行为, 但最终的决定权还是在 被提醒人自己。同时从该单词的本义可以看出, 助推 理论是不采用明显的禁止或经济刺激方式, 但却能有 效影响人们行为并朝向设计好的或可预见的方向发 展的选择架构导向。近年来, 越来越多的专家学者开 始关注该理论, 但截止目前, 国内绝大多数关于助推 理论的文章主要集中在研究助推类型的归纳, 缺乏对 该理论应用的梳理。相对而言, 国外学者对助推理论 的研究相对较为广泛和深刻, 从食品学到经济学到管 理学甚至到公共政策等领域都受到学者们的广泛关 注。近年来因为经济形势的变化, 在营销学领域, 如 何利用助推理论影响消费者心理, 满足消费者需求, 从而影响消费行为受到越来越多经济学家的关注。

\section{1. 由心而制}

经济结构不断优化和调整使市场经济不断完善, 与此同时, 市场中买卖双方的角色和地位也在不断发 生调整与变化。全球市场快速由“产品导向”、“销售 导向”转变为“顾客导向”。各个企业都清晰地意识到, 只有提供让顾客满意的商品及服务, 才能真正有效地 形成自己的优势，在激烈的

市场竞争中站稳脚跟。因此在企业发展的过程中, 越来越多的管理者开始重视顾客的体验感, 极尽可能 的通过各种办法提升消费者的服务体验从而吸引顾 客, 挽留他们的忠诚并提升他们的满足感。在此前提 下, 助推理论受到了越来越多人的关注, 他们普遍认 为助推理论本质上是鼓励或指引人们行为的方式, 这 种方式不存在任何命令或强迫, 也不需要很强的经济 激励或处罚, 而是自主性的选择。Hollands 等曾经也 指出助推是通过改变微观环境中对象或刺激的属性 或物品的摆放来影响、引导人的健康行为。简单地说, 助推理论是以小博大, 通过看似不经意的举措进行有 目的地干预, 使人们在自由的心理状态下做出更好的 选择。

所以近年来社会型助推成为很多企业备受追捧的 方式: 即通过传递他人的行为规范可以有效影响周边 人的选择。这一观点在学术界也得到了证实, 普遍认 为该方式可以有效干预消费者选择。因为人们对于权 威或关系亲近的人会产生一种莫名的信任, 因此会更 倾向从他们那里获取信息, 他们的选择也具有更高的 可信度, 因此消费者容易受这些信息的影响和引导而 做出选择。对消费者而言“大多数人的选择”, “我朋 友用了”，“他说的应该没错”等等的心理暗示都会对 他们的选择产生微妙且直接的影响。这种看似漫不经 心的影响不仅会影响消费者的购买决策, 还会让他们 在购买过程中感觉买的很踏实、很放心。因此这无形 中就提升了产品使用中的愉悦感。

另外, 消费者还有一种很常见的心理: 嫌恶损失, 他们普遍讨厌损失本来属于自己的东西。再小, 再不
值钱, 但凡失去某样东西, 他们难过的程度远远比得 到它所获得的快乐的程度大得多。曾经有一个实验, 给罐头加上“每人限量 12 罐”的标语, 很多路过的顾 客都觉得这是一笔不容错过的划算交易, 不多买点就 亏大了, 抱着这种占大便宜的心理, 顾客在购物车里 放入更多的罐头。最终每位消费者从平均购买 3.3 罐增加到平均购买 7 罐。因此商家常见的限时特惠、 聚划算、会员专享等优惠信息都无形中影响着消费者 的决策过程。不仅激励顾客下单, 有效地促进了消费, 同时还无形中让消费者极大地获得了满足感。这一举 措在促进消费的同时, 还一定程度上促使普通消费者 转化为会员，甚至是付费会员。

由此可见，商家在设计营销活动时应该充分了解 消费者的心理规律和特征从而进行设计, 只有走心的 设计, 才能在无形中引导消费者的决策过程, 提升他 们的满意度和忠诚度。

\section{2. 人性化氛围}

正如前面所讲, 助推理论有着明显的经济学的目 的, 试图通过助推改变人们的消费动机和行为从而影 响人们的决策过程, 所以助推理论的应用是为了帮助 设计师设计出更完美的选择系统, 从而更自然地引导 人们做出设计者所期望的选择。

助推理论的作者曾经指出人具有两套思维系统, 理性思维系统和直觉思维系统。通常情况下, 大部分 人在做选择时往往依赖直觉思维系统, 因此非理性的 盲目选择在生活中随处可见。当今市场的很多营销方 案正是利用了消费者的这一特点。例如, 近年来会员 制营销比比皆是, 很多消费者甚至开始接受付费会员 制因为可以享受到更多的福利和更好的服务。各个 企业也制定了属于自己的会员日。会员日当天购买 所有商品都可享受一定的价格优惠或积分翻倍等福 利。例如盒马鲜生, 一家集超市, 餐饮, 菜市场功能 于一体的新型的零售店最初选定了每周二为会员日, 当天所有商品, 包括打折商品, 付费会员都可享受 8.8 折优惠, 此举极大地刺激了消费者的购买欲。2020 年，企业更是升级了会员服务，付费会员日不再是固 定的每周二，而是客户可以根据自己的时间自主选 择周二或周三，虽然两个都是工作日，但这个小小 的改变, 却给消费者带来了更大的方便, 让他们有 了选择的满足感, 也极大地提升了企业的口碑和知名 度。另外盒马在方便冷冻菜肴的冷柜上方安装了烹 杄这些产品的教学视频。几乎没有声音, 所以并不嘈 杂，但烹杄时菜有的色、香、味透过清晰的画面让很 多消费者驻足, 加上旁边熟食区传来的阵阵香味都让 很多路过的消费者在没有计划的前提下加购了该类 商品。因此人性化的消费环境可以使消费者在进行选 择和消费时感觉更舒服，在没有任何被强迫的状态下 做出超出理性的购买行为。

虽然助推不一定每次都会直接改变人们的选择, 但它确实通过有目的的干预和诱导, 借助巧妙的设计 在尊重人们选择自由的前提下帮助他们做出更好的 
选择，从而帮助企业实现特定目标。综上所述，助推 理论是一种观念, 一种看似漫不经心的提醒, 一种潜 意识里的影响, 在保留人们选择自由的同时, 无形中 引导人们朝向更好的方向行动。

\section{3. 便捷安全}

《第 46 次中国互联网络发展状况统计报告》的最 新数据显示, 截至 2020 年 6 月, 我国网民规模达 9.40 亿，较上季度增长 3625 万，互联网普及率达 $67.0 \%$, 较 2020 年 3 月提升 2.5 个百分点。截止 2020 年 6 月, 我国网络购物用户规模达到 7.49 亿，较 2020 年 3 月增长 3912 万, 手机网络购物用户达到 7.47 亿, 较 2020 年 3 月增长 3947 万。由此可见, 我国网购 的消费者数量呈现稳步增长趋势。蓬勃发展的互联网 购物直接改变了消费者的购物习惯和购物观念。

但网购给消费者带来巨大方便的同时，也带来了 一些麻烦: 当输入想要购买的产品时, 几万甚至几十 万件产品会瞬间蹦出来, 这时从无穷尽的商品中选出 自己想要的实在是一件费时费力且让人头疼的事情。 即便可以通过网站的销量、价格、信用等因素的排序 来帮助自己, 但面对如此多的同类产品, 篮选貌似没 多大作用, 这极大地削弱了消费者的满足感。在此情 况下, 多家网购平台都推出精准推荐服务。平台根据 消费者在网页上的浏览痕迹及购物评价来分析消费 者的购物喜好帮助他们篮选产品, 这不仅为消费者的 网购提供了极大的方便, 也提高了网站的吸引力和市 场竞争力。

但精准推荐给消费者购物带来了极大方便的同时 也带来了一些潜在的麻烦, 让关注个人隐私的消费者 感到危机, 因为精准推荐是在消费者不知情的情况下 记录了他们网页汶览痕迹、点击次数、商品评价等隐 藏数据。

助推理论的应用给设计师带来了灵感，启发他们 从用户需求出发, 设计出对用户更友好的选择环境和 选择体系。针对隐私的问题, 越来越多的网站在会员 服务中通过增加一个选项提供了不留下任何汶览记 录的服务, 这给消费者带来了极大的安全感, 也提升 了他们的满意度和忠诚度。例如 Netflix, 美国著名的 视频网站之一, 该平台用户将看过的电影归纳整理成 影单, 平台收集这些喜好, 给该用户推荐与其品味相 同的电影爱好者的影单, 这种协作式篮选对于用户发 现新领域新知识有很大的促进作用, 也让公司在市场 有了很好的口碑。同时考虑到顾客隐私, 公司近年来 推出了一个隐私观看模式, 对观看记录进行绝对保 密。这意味推荐时隐私模式下的数据不会被采纳。这 一贴心举措完美解决了很多客户的顾虑, 让该网站变 得安全可靠, 因此极大地提升了在消费者心目中的口 碑。

由此可见, 助推理论是针对人的行为进行助推, 通过助推有意识地影响和改变而不是直接强加公司 的意志在消费者身上。该理论的应用有效引导设计师
设计出更友好, 更人性化、更体贴的选择环境, 继而 提升消费者的满意度和忠诚度。

\section{4. 尊重消费者}

综上所述, 助推理论的核心内容是从用户出发, 了解用户。从用户角度出发帮助设计者设计出对用户 更友好的选择环境。

近年来，随着经济的不断发展，付费服务越来越 多，在享受更完善服务的同时，相信很多消费者都有 过类似的经历，在自己遗忘的情况下，平台会自动完 成续费。这种“方便”让很多消费者常常因为忙碌而忽 略它的存在; 有些则因为忘记取消续订而暗暗损失了 很多钱, 因此抱怨自己受骗, 继而也对平台产生了失 望的情绪; 有些法律意识强的用户甚至和平台打起了 官司。虽然自动扣费对平台来说是一种有效的盈利方 式, 但却严重影响了用户的体验感, 甚至会让用户有 受骗的感觉，这对企业的形象没有一点好处，甚至会 让多年苦心经营的口碑瞬间崩塌。很多企业已经意识 到这个问题的严重性，因此会通过多种方式提醒用 户，让他们自行决定是续费还是取消续订。这类信息 的确认方式通常都很简单, 一秒就可以完成。很多企 业更是巧妙的将续费设为默认选项，有些消费者用惯 了或者图方便瞄一眼直接点确认键。

即便消费者看似如此随意地做出了选择, 但让他 们对自己的付费行为有所了解而做出抉择，而不是平 台暗地里默默扣除用户的费用, 这一行为有效提升企 业的品牌形象和巩固会员的忠诚度。由此可见, 设计 者设计的选择体系应该体现对用户行为的深刻理解, 要保证人们的直觉思维不受到迷惑, 心甘情愿地在巧 妙设计中做出更好的选择。这样既尊重了消费者的选 择权又为企业省去了很多麻烦。

\section{5. 优雅的反馈}

了解消费者的偏好，善待消费者，除了从消费者 角度出发, 还应开通和完善有效的反馈体系。很多知 名企业都意识到，正确对待用户反馈会为企业带来无 限的资源。例如早期数码相机拍照后没有提示音, 很 多人都不知道到底拍摄成功与否，因此经常出现没 拍上或多张重复照片的情况。后来听取用户反馈, 加 入了提示音，极大地提升了用户体验感。

由此可见, 完善的反馈体系非常重要, 因此一定 要保证反馈渠道的畅通性, 使企业能够及时收到用户 的感受反馈，而用户也可以及时获取到企业的体贴 关心。例如近年来, 人们的生活水平越来越高, 越 来越多的人已跻身肥胖行列, 因此有关健康的话题 得到了越来越多人的关注, 除了注意饮食, 很多人 会选择到专业的健身中心进行锻炼。但健身并不是一 个轻松的过程。因此适时、适量地反馈有利于鼓励消 费者。在有的健身中心, 跑步机上显示热量消耗表, 这样消费者就不用等到三四天后上体重机时才能看 到自己的变化, 而是直接可以看到消耗的卡路里。这 
远比教练的语言反馈更直接、更有效。在买跑步机或 进健身房时, 消费者可能并没有这么具体的要求，但 这一细心的设计让健身消费者有了及时且隐私的反 馈, 从而直接刺激了他们的热情, 也成为他们持续坚 持运动的动力。

另外, 现在很多企业都有电话回访等服务用以提 升产品和服务的质量, 提高顾客的留存率, 但反馈的 频次和方式非常关键, 过于频繁或过于模式化都很容 易让用户反感或产生无感, 故而无法发挥反馈的作 用。因此反馈不要太过于频繁, 也不要制式问答。要 让消费者觉得是被关心而不是被打扰或者盯上了, 太 过于频繁的回访往往会让消费者产生反感, 反而不能 发挥良好的作用。因此企业在设计时, 要了解消费 者心理和需求, 要精心的, 优雅的, 体贴的根据对消 费者的了解设计内容和方式来进行有效沟通。

\section{6. 结论}

总而言之, 经济的发展让市场由产品导向和商品 导向迈向消费者导向。顾客的满意度直接决定了企业 的命运。提高顾客的满意度和忠诚度, 降低顾客的转 移率直接导致企业销售额的大幅提高。忠诚的顾客不 仅会选择长期购买企业的产品和服务, 还会主动成为 企业的宣传大使, 从而影响身边人的购买行为, 这无 形为企业带来极大的竞争优势。助推理论可以基于对 消费者需求了解的基础上做出很多精心的小设计从 而有效提升顾客忠诚度, 它既是有效的营销手段又是 提升用户满意度的有效设计, 通过控制人们在认知和 动机上某种缺陷和不足, 修复和完善在特定情境下的 自主偏差, 让消费者在自由意识的基础上做出更好的 选择。

\section{REFERENCES}

[1] Halpern D. D., Inside the Nudge Unit: How Small Changes Can Make a Big Difference[J], International Journal of Market Research, 2015, 58 (1), pp. 155-157.

[2] Hollands G. J., Shemilt I., Marteau T. M., et al., Altering Micro-Environments to Change Population Health Behaviour: Towards an Evidence Base for Choice Architecture Interventions[J], BMC Public Health, 2013, 13(1), 1218, pp. 1-6.

[3] Le Zhi, The Power of Words[J], QunZhong, 2018, 4, pp.60.

[4] Robinson E., Fleming A., Higgs S., Prompting Healthier Eating: Testing the Use of Health and Social Norm Based Messages[J], Health Psychology, 2014, 33( 9), pp. 1057-1064.

[5] Wilson A. L., Buckley E., Buckley J. D., et al., Nudging Healthier Food and Beverage Choices through Salience and Priming. Evi-dence from a
Systematic Review[J], Food Quality and Preference,2016,51,pp. 47-64. 